\title{
THE MICROWAVE ELECTRON LINAC IN THE TREATMENT OF CANCER
}

\author{
John C. Ford, PhD - Varian Medical Systems \\ Palo Alto, CA USA
}

\begin{abstract}
Immediately after the discovery of X-rays at the end of the nineteenth century the enormous medical value of ionizing radiation was clear. Radiation has certain unique properties for cancer treatment not duplicated by any other modality. In the modern clinical setting more than half of all cancer patients are treated with radiation and this percentage is increasing with the availability of new techniques. X-rays dominate with electrons used in 5 to 10 percent of the cases and other kinds of radiation playing a peripheral role. An extraordinary effort went into the search for the optimum radiation treatment machine during the twentieth century. Microwave electron linacs producing bremsstralung beams of 4 to 6 megavolts and versions producing bremsstralung beams from 4 to $25 \mathrm{MV}$, with ancillary electron beams from 4 to $20 \mathrm{MeV}$, have emerged as the overwhelming treatment system of choice. Today these machines are computer controlled and can provide advanced dose shaping capabilities with the use of dynamic Multi-Leaf Collimators, MLC's in conjunction with specialized dose planning systems. WHO statistics indicate a doubling of cancer incidence and mortality by the year 2020 and an increasing need for such equipment. Commercially available medical linacs are impressively robust and extraordinarily cost effective and are used extensively in both developed and developing countries.
\end{abstract}

\section{MEDICAL LINACS}

The biggest application of the microwave electron linac has been in the worldwide fight against cancer.There is no accurate official count of medical linacs in operation today but the number is on the order of 7500 , with approximately half in the United Sates. Many of these existing machines are old and need to be replaced and there is great need for new treatment facilities therefore there is an enormous deficit of medical linacs today. It is estimated that 14 million people fall victim to cancer annually, and ideally about 60 percent should receive radiation treatment as either the primary treatment or as an adjunct treatment to surgery or chemotherapy. The cancer incidence rate will increase by twice in the next 20 years. The cancer incidence rate is much higher in

the industrialized nations, fundamentally due to the greater age of these populations. However, cancer is a significant problem in the developing world based simply on the larger size of these populations. The WHO estimates that within twenty years cancer will be the leading cause of death everywhere with the exception of sub-Saharan Africa. Today the death rate from cancer is about 7 million per year and this number could be reduced significantly by the greater availability of medical linacs, as well as the new treatment techniques available with these modern machines. Also, of very great importance is the role medical linacs play in palliative therapy to reduce the suffering of terminal cancer patients.

Medical linacs have been highly successful because they produce X-ray and electron beams at clinically useful energies, at relatively low cost, and with high patient throughput. These machines are impressive in their reliabilty and they are quite robust with very long operational lifetimes. For example, one such medical linac using a vertically mounted standing wave accelerator has been operating reliably with high patient loads in South America for more than thirty years. Modern machines are very compact and can rotate 360 degrees isocentrically. They have numerous ancillary systems such as precise patient couches, beam modifying systems, high energy beam imaging, and computer network interfaces.
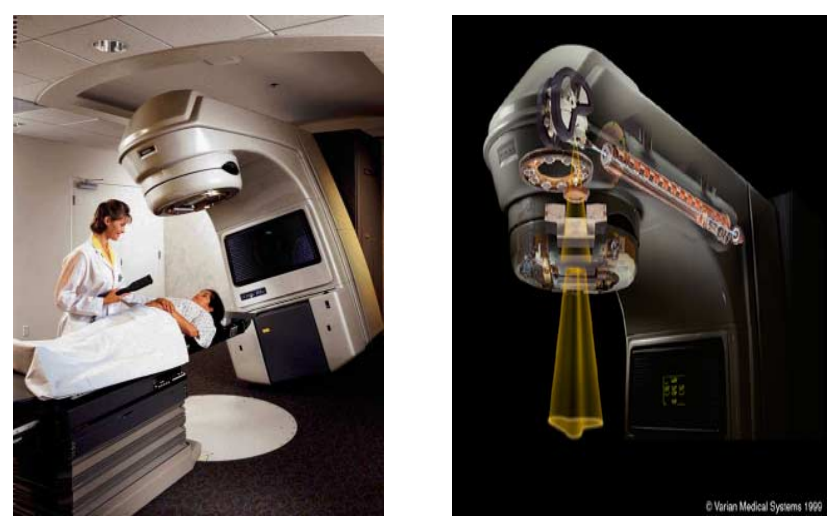

On the left a modern medical linac is shown. On the right the accelerator structure, bend magnet, and standard collimators with MLC, and beam are visible. Photographs courtesy of Varian Medical Systems. All rights reserved.

\section{RATIONALE FOR RADIOTHERAPY}

The treatment of cancer by radiation was reported more than 100 years ago and today has developed into one of the most successful and advanced medical specialties. The value of radiation as a therapeutic modality is clear. Cells have evolved in an environment where radiation, as well as chemical, stress is ever-present. Consequently, cells have developed impressive repair mechanisms. Cancer cells are typically more radiosensitive than normal cells as they have impaired repair mechanisms. This provides for a therapeutic strategy whereby radiation is delivered to a 
tumor by dose fractions, which steadily attenuate the cancer cells. The size and timing of the fractions allow the normal cells to repopulate and replace the diseased cells. Success depends on achieving a sufficient 'tumorcidal dose' that does not sacrifice too much normal tissue, which is unable to repair itself. The ratio of diseased tissue destroyed to normal tissue sacrificed is called the 'therapeutic ratio.' It is a complicated function of many variables, some of which are not well understood. But numerous treatment regimes have evolved over many years of clinical practice that achieve sufficient therapeutic ratio. They all depend on knowing the magnitude of the dose delivered, distributing the dose spatially in a planned fashion and fractionating the dose properly. The probability of controlling (clinically eradicating) a tumor is a function of total dose with sigmoid shaped curve. The probability of causing 'unacceptable morbidity' due to the destruction of too much normal tissue is a similarly shaped curved which is fortunately translated to the right of the 'tumor control' curve.

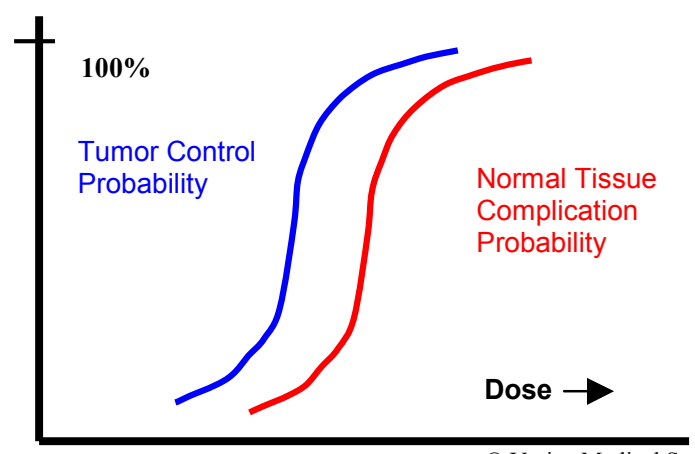

(C) Varian Medical Systems 2001

The success of treatment depends on finding an achievable therapeutic ratio, moving as high on the tumor control curve as one can while moving up the morbidity curve as far as one dares. There is really a complex of morbidity curves, and different tumor control curves for different regions of tissue, which somehow have to be collapsed into a single overall relationship guiding the selection of the total dose, its spatial distribution and the fractionation. Thus, in actual practice, a great deal of skill, experience and intuition is required of the 'radiation oncologist'. Radiation therapy is actually delivered by a team of professionals, which includes medical physicists. Modern techniques have enhanced the role of the medical physicist, as did the linac thirty years ago.

The beauty of radiation, especially as delivered by the medical linac, is that it is highly quantitative in terms of dose delivery and can be delivered in precisely known doses with the desired spatial distribution and fractionation. This is critical because the number of diseased cells in a given region determines the total dose required to achieve a given probability of control. On the other hand, the various types of normal cells, with varied sensitivity, which must be preserved in a given region determines the maximum dose possible. All this must be considered along with an optimum scheme of fractionation selected by taking into account such nonbiological considerations as cost and machine availability. Radiation can be highly localized or distributed as a regional treatment, which gives it a power not available to surgery or chemotherapy.

\section{ADVANCED RADIOTHERAPY}

During the last twenty years advances in medical imaging have set the stage for a revolution in radiotherapy. Computed Tomograpy Scanners, CT, have allowed for accurate, morphologically correct, threedimensional anatomical imaging. These CT images are really three dimensional electron density maps ideal for the calculation of radiation dose absorbed from X-ray and electron beams. Magnetic Resonance Imaging, MRI, has played a role as well. Today, there is much excitement with Positron Emission Tomograpy, PET, imaging, especially when fused with CT images, because this allows some inferences to be drawn with respect to disease cell distribution and possibly the changes in the tumor during the course of therapy. Three-dimensional, volumetric, imaging makes it possible to define specific treatment volumes. It is then possible to design a treatment where particular levels of radiation dose are delivered to these volumes as a function of the fractionation scheme. This is the aim of 'conformal' radiotherapy. The beam can also be time-gated to account for organ/tumor motion.

Rather elegant conformal dose distributions can be achieved by a new technique known as Intensity Modulated Radiation Therapy, IMRT. The newest generation of medical linacs allows for the precise computer control of the radiation beam as a function of the machine axes (i.e. mechanical degrees of freedom). Multi-Leaf Collimators, MLC's, have been developed that now allow for X-ray beams with irregular shapes. Beams of different shapes can be delivered at different dose levels and at different beam directions. Shaped beams from several directions can thus be combined to produce a desired, pre-planned, three-dimensional, conformal dose distribution.

Some advanced MLC's have leaves that can move rapidly across the beam field in a dynamic control mode. Opposing leaves can form a 'sliding window' with a width that varies under computer control. Each leaf pair can have its own trajectory to give a particular radiation flux profile. As many as 60 leaf pairs can form a radiation field. This type of MLC can produce high resolution shaped beams as fast as conventional open fields, and faster than fields shaped with absorbing filters that are very difficult to fabricate and time consuming to use.

In radiotherapy, the therapeutic ratio can be increased by escalating the tumor dose while offsetting tissue morbidity with better dose conformation. IMRT has already produced some dramatic dose escalations in the treatment of prostate cancer while actually reducing morbidity. The data indicate that this is leading to 
improved survival rates. IMRT is now being used with a number of other forms of cancer.

The implementation of IMRT requires the use of other technology for determining the conformal dose distributions and the subsequent calculation of the MLC leaf trajectories. With conventional therapy the beam weights (dose per beam), field size and beam direction are proposed based on clinical experience then the resulting dose distribution is calculated by computer programs. A few iterations are made to obtain a more desirable dose distribution. This is the so-called conventional Forward Treatment Planning process.

IMRT has ushered in a new approach and a technique called Inverse Treatment Planning. Here the dose distribution is described by a set of macro-clinical parameters, which includes the number and direction of the beams, regions to be protected and volumes to reach a minimum tumorcidal dose. Then an Inverse Planning computer algorithm determines the beam shape and beam weights. These algorithms utilize various multidimensional optimization techniques such as synthetic annealing and the conjugate gradient method. Once the beam shapes are known the leaf trajectories can be calculated and from this information and from the beam weights, the IMRT treatment can be determined.

\section{THE FUTURE}

On the horizon is true 'bio-effect' planning, not simply dose planning, and perhaps 'adaptive radiotherapy' where the overall fractionation scheme will be changed during the course of treatment, as a function of image assessment of the tumor, and perhaps cell assay and other biological markers. All this information will be collated, correlated, planned and optimized and communicated to the medical linac via its departmental network interface.

There is a view that advances in biotechnology might some day render radiotherapy obsolete. However there is a contrary view that these advances will lead to ways to actually manipulate cellular radiosensitivity that will then increase the value of radiation treatment many fold. Certainly, the future is bright for the next twenty years, or more, even within the confines of conventional radiation therapy technology, simply given the typical clinical presentation of the disease and the epidemiology of the worldwide cancer problem.

There are competitors to the medical linac. Most notably, at the high-cost end of the spectrum, is the proton accelerator. High energy proton beams (from 240 to 270 $\mathrm{MeV}$ ) give clinical beams with well defined Bragg peaks and highly conformal dose distributions. However, the present generation of medical linacs give practical IMRT conformal dose distributions with X-rays that are arguably as good as protons. This issue revolves around the clinical significance of dose gradient and lower level dose volumes. This is not so straightforward because cancer cells are never that well localized, and also within a clinical context a dose below a certain level may be effectively equivalent to zero dose. But, the biggest issue with proton machines is shear cost that can easily be more than 30 times that of a linac. Given this, and the facility cost, proton accelerators provide no global solution to treating cancer, though they may make an important contribution in certain large treatment center and university settings. 